\title{
Thermal Casimir-Polder interaction of different atoms with graphene
}

\author{
M. Chaichian, ${ }^{1}$ G. L. Klimchitskaya,${ }^{2}$ V. M. Mostepanenko, ${ }^{2}$ and A. Tureanu ${ }^{1}$ \\ ${ }^{1}$ Department of Physics, University of Helsinki, \\ P.O. Box 64, FIN-00014, Helsinki, Finland \\ ${ }^{2}$ Central Astronomical Observatory at Pulkovo of the \\ Russian Academy of Sciences, St.Petersburg, 196140, Russia
}

\begin{abstract}
The thermal correction to the energy of Casimir-Polder interaction of atoms with a suspended graphene membrane described by the Dirac model is investigated. We show that a major impact on the thermal correction is made by the size of the gap in the energy spectrum of graphene quasiparticles. Specifically, if the temperature is much smaller than the gap parameter (alternatively, larger or of the order of the gap parameter), the thermal correction is shown to be relatively small (alternatively, large). We have calculated the free energy of the thermal Casimir-Polder interaction of atoms of $\mathrm{He}^{*}, \mathrm{Na}, \mathrm{Rb}$, and $\mathrm{Cs}$ with graphene described by both the hydrodynamic and Dirac models. It is shown that in exact computations using the Dirac model, one should use the polarization operator at nonzero temperature. The computational results for the Casimir-Polder free energy obtained in the framework of hydrodynamic model of graphene are several times larger than in the Dirac model within the separation region below $2 \mu \mathrm{m}$. We conclude that the theoretical predictions following from the two models can be reliably discriminated in experiments on quantum reflection of different atoms on graphene.

PACS numbers: $31.30 . j \mathrm{~h}, 34.35 .+\mathrm{a}, 12.20 .-\mathrm{m}, 42.50 . \mathrm{Ct}$
\end{abstract}




\section{INTRODUCTION}

The Casimir-Polder force acting between a microparticle and a macrobody is caused by the existence of quantum fluctuations of the electromagnetic field. This phenomenon is a manifestation of the more general dispersion forces at relatively large separations where the retardation of the electromagnetic interaction becomes significant (at separations below a few nanometers the electromagnetic interaction can be considered as instantaneous and the dispersion force is usually referred to as the van der Waals force). Quantum theories of the van der Waals and Casimir-Polder forces were developed by London [1] and (for the case of an atom interacting with an ideal metal plate) by Casimir and Polder [2], respectively. For an atom interacting with a plate made of some real material described by the frequencydependent dielectric permittivity, the unified theory of the van der Waals and Casimir-Polder forces was developed by Lifshitz [3] (see also monographs [4 6] $)$.

Nowadays, the Casimir-Polder interaction between different atoms and material plates (cavity walls) has attracted much attention in experiments on quantum reflection [7-9]. This is a process in which an ultracold atom is reflected by an attractive atom-wall potential or, in other words, it is an above-barrier reflection. It was shown [10] that quantum reflection is very sensitive to the specific form of the Casimir-Polder interaction. Theoretically, calculations of the Casimir-Polder forces between various atoms and plates made of different materials were performed [11-14] on the basis of the Lifshitz theory. The results obtained were compared [15] with those calculated using a simple phenomenological potential [8, 16, 17].

Recently, special attention has been directed to the Casimir-Polder interaction with carbon nanostructures, such as graphene, carbon nanotubes, and fullerenes [18]. Computations were performed using the phenomenological density-functional approach [19-22], secondorder perturbation theory [23] and, for multiwalled carbon nanotubes with at least several walls, using the Lifshitz theory [24]. For one-atom-thick carbon nanostructures, the concept of dielectric permittivity is not immediately applicable (it becomes applicable, for example, to thin fullerene films and can be used to deduce the dynamic polarizabilities of single fullerene molecules [25]). In order to extend the Lifshitz theory to this case, the reflection coefficients of electromagnetic oscillations on graphene were found using the hydrodynamic model [26, 27]. For this purpose, graphene was treated as an infinitesimally thin positively 
charged flat sheet, carring a homogeneous fluid with some mass and negative charge densities. The obtained reflection coefficients depend on temperature only through the Matsubara frequencies. Calculations of the Casimir-Polder interaction in the framework of the hydrodynamic model are presented in Refs. [28, 29] (see also [6, 30]).

The hydrodynamic model of graphene is only a crude approximation. It does not take into consideration that low-energy excitations in graphene are massless Dirac fermions except for the fact that they move with a Fermi velocity, rather than with the speed of light [18, 31]. From this, it follows that at low energies the dispersion relation for quasiparticles is linear with respect to the momentum measured relative to the corner of the graphene Brillouin zone. The Dirac model of graphene assumes that the dispersion relation is linear at any energy. Using this assumption, the corresponding reflection coefficients for the electromagnetic oscillations at zero temperature have been found [32] different from those obtained with the hydrodynamic model. The obtained coefficients depend on the polarization operator in an external electromagnetic field calculated in the one-loop approximation in three-dimensional spacetime. Note that the exact value of the gap parameter $\Delta$ of quasiparticle excitations entering the polarization operator is not known. In Ref. [33] it was shown that Dirac model leads to much smaller values of the van der Waals (Casimir-Polder) atom-graphene interaction than the hydrodynamic model at separations below $100 \mathrm{~nm}$. Keeping in mind the precision achieved in experiments on quantum reflection of ultracold atoms [7 -9, 34, 35], it was concluded that it is possible to experimentally distinguish between the predictions of the Dirac and hydrodynamic models of graphene.

The thermal effect in the Casimir and Casimir-Polder interactions is a subject of debate [6, 30]. For the thermal interaction between two graphene sheets it was argued [36] that the thermal regime starts at rather low temperatures $T$ (or, respectively, short separation distances of tens of nanometers at room temperature) because the value of the effective temperature is determined by the Fermi velocity $v_{F}$ rather than the speed of light $c$. This conclusion was, strictly speaking, obtained in a nonretarded regime. A fully consistent quantum-field version of the Dirac model at nonzero temperature was presented in Ref. [37]. In that paper the temperature-dependent polarization operator and respective coefficients for electromagnetic oscillations on graphene at nonzero temperature were derived. Computations were performed for a graphene layer with the gap parameter equal to zero interacting with a metallic plate. For this system the conclusion of Ref. [36] on the existence of large 
thermal corrections at short separation distances was qualitatively confirmed (with a proviso that the characteristic distance separating zero- and high-temperature regimes depends on the fine-structure constant rather than on the Fermi velocity). In Ref. [38] quantum reflection of ultracold atoms from thin films, semiconductor heterostructures and graphene was considered at $T \neq 0$ with graphene being described by both the hydrodynamic and Dirac models. It was concluded that suspended graphene membranes produce higher quantumreflection probabilities than bulk matter. The remark made earlier [33] that already achieved experimental precision allows discrimination between the predictions of different models of graphene was supported. This conclusion was obtained using the polarization operator at zero temperature [32]. Keeping in mind that suspended graphene membranes with rather large diameter (of $55 \mu \mathrm{m}$ ) are already available [39], it seems pertinent to perform a full quantum-field theoretical investigation of the thermal Casimir-Polder force between various atoms and such membranes in the framework of different models of graphene proposed in the literature.

In this paper, we calculate the free energy of the thermal Casimir-Polder interaction between atoms of metastable helium $\left(\mathrm{He}^{*}\right)$, sodium $(\mathrm{Na})$, rubidium $(\mathrm{Rb})$, and cesium $(\mathrm{Cs})$ and a suspended graphene sheet. Graphene is described using either the hydrodynamic model or the Dirac model and the obtained results are compared. In the framework of the Dirac model, the polarization operator at nonzero temperature is employed and the dependences on the value of the gap parameter $\Delta$ are investigated. We demonstrate that under the condition $k_{B} T \ll \Delta$, where $k_{B}$ is the Boltzmann constant, the thermal correction is relatively small, but if $\Delta \lesssim k_{B} T$ there are large thermal corrections to the Casimir-Polder force. At the same time, the magnitude of the thermal correction strongly depends on the atom-graphene separation. The value of the Fermi velocity is shown to be of no crucial influence on the magnitude of thermal correction for atom-graphene system. The novelty of this paper is that the interaction of different atoms with graphene was investigated using the full Dirac model at nonzero temperature with the temperature-dependent polarization operator. In so doing it was found that the relative size of thermal correction strongly depends on the gap parameter. Our computations show that experiments on quantum reflection are capable to discriminate between the predictions of the hydrodynamic and Dirac models of graphene. One can also conclude that in the framework of the Dirac model it is necessary to take into account the dependence of the polarization operator on temperature. According to our 
results, the use of the zero-temperature polarization operator in computations at $T=300 \mathrm{~K}$ can lead to large errors in theoretical predictions for some values of parameters.

The paper is organized as follows. In Sec. II we present a brief formulation of the two models of graphene, introduce the reflection coefficients and illustrate the limiting case of zero temperature for the Dirac model. Section III contains the investigation of dependences

of the thermal correction to the Casimir-Polder energy on the gap parameter. In Sec. IV the distance dependences of the Casimir-Polder interaction of different atoms used in experiments on quantum reflection with graphene are calculated. In Sec. V the reader will find our conclusions and discussion.

\section{TWO DIFFERENT MODELS FOR THE REFLECTION COEFFICIENTS ON GRAPHENE}

The unified expression for the van der Waals and Casimir-Polder free energy of an atom interacting with a planar structure is given by the Lifshitz formula [3 6 ]. This formula can be expressed in terms of reflection coefficients of the electromagnetic oscillations on this structure (in our case on graphene) in the following way:

$$
\begin{aligned}
& \mathcal{F}(a, T)=-\frac{k_{B} T}{8 a^{3}} \sum_{l=0}^{\infty} \alpha\left(i \zeta_{l} \omega_{c}\right) \int_{\zeta_{l}}^{\infty} d y e^{-y} \\
& \quad \times\left\{2 y^{2} r_{\mathrm{TM}}\left(i \zeta_{l}, y\right)-\zeta_{l}^{2}\left[r_{\mathrm{TM}}\left(i \zeta_{l}, y\right)+r_{\mathrm{TE}}\left(i \zeta_{l}, y\right)\right]\right\}
\end{aligned}
$$

Here, $a$ is the separation distance between the atom and the graphene sheet, $\alpha\left(i \xi_{l}\right)$ is the dynamic polarizability of an atom calculated along the imaginary Matsubara frequencies $\xi_{l}=$ $2 \pi k_{B} T l / \hbar$ with $l=0,1,2, \ldots$, the dimensionless Matsubara frequencies are $\zeta_{l}=\xi_{l} / \omega_{c}$, the characteristic frequency is defined as $\omega_{c}=c /(2 a)$, and $r_{\mathrm{TM}, \mathrm{TE}}$ are the reflection coefficients for two independent polarizations of the electromagnetic field (transverse magnetic and transverse electric, respectively). The prime near the summation sign multiplies the term with $l=0$ by $1 / 2$. Note that the dimensionless frequencies $\zeta_{l}$ are functions of the separation $a$. 


\section{A. Hydrodynamic and Dirac models of graphene}

As was discussed in Sec. I, there exist two different models of reflection coefficients for graphene. In the framework of the hydrodynamic model the reflection coefficients take the form [26 28]

$$
\begin{aligned}
& r_{\mathrm{TM}}\left(i \zeta_{l}, y\right) \equiv r_{\mathrm{TM}}^{(h)}\left(i \zeta_{l}, y\right)=\frac{\tilde{K} y}{\tilde{K} y+\zeta_{l}^{2}}, \\
& r_{\mathrm{TE}}\left(i \zeta_{l}, y\right) \equiv r_{\mathrm{TE}}^{(h)}\left(i \zeta_{l}, y\right)=-\frac{\tilde{K}}{\tilde{K}+y} .
\end{aligned}
$$

Here, $\tilde{K}=2 a K$ and $K=6.75 \times 10^{5} \mathrm{~m}^{-1}$ is the characteristic wave number of the graphene sheet which corresponds to the frequency $\omega_{K}=c K=2.02 \times 10^{14} \mathrm{rad} / \mathrm{s}$. The dimensionless variable $y$ is connected with the projection of the wave vector on the graphene sheet $k_{\perp}$ by the equation $y=2 a\left(k_{\perp}^{2}+\xi_{l}^{2} / c^{2}\right)^{1 / 2}$. As was mentioned in Sec. I, the hydrodynamic model does not take into consideration that the dispersion relation for quasiparticles in graphene is linear with respect to momentum. It should be mentioned also that the parameter $K$ in the hydrodynamic reflection coefficients (2) is temperature-independent.

In the framework of the Dirac model the reflection coefficients are expressed in terms of the components of the polarization tensor [37]

$$
\begin{aligned}
r_{\mathrm{TM}}\left(i \zeta_{l}, y\right) & =\frac{y \tilde{\Pi}_{00}}{y \tilde{\Pi}_{00}+2\left(y^{2}-\zeta_{l}^{2}\right)}, \\
r_{\mathrm{TE}}\left(i \zeta_{l}, y\right) & =-\frac{\left(y^{2}-\zeta_{l}^{2}\right) \tilde{\Pi}_{t r}-y^{2} \tilde{\Pi}_{00}}{\left(y^{2}-\zeta_{l}^{2}\right)\left(\tilde{\Pi}_{t r}+2 y\right)-y^{2} \tilde{\Pi}_{00}},
\end{aligned}
$$

where the dimensionless components $\tilde{\Pi}_{00, t r}$ are connected with the dimensional $\Pi_{00, t r}$ by the equation $\tilde{\Pi}_{00, t r}\left(i \zeta_{l}, y\right)=(2 a / \hbar) \Pi_{00, t r}\left(i \zeta_{l}, y\right)$. The explicit expressions for the components of the polarization operator at nonzero temperature were obtained in Ref. [37]. In terms of our dimensionless variables the component $\tilde{\Pi}_{00}$ can be identically represented in the following 
form:

$$
\begin{aligned}
& \tilde{\Pi}_{00}\left(i \zeta_{l}, y\right)=8 \alpha\left(y^{2}-\zeta_{l}^{2}\right) \int_{0}^{1} d x \frac{x(1-x)}{\left[\tilde{\Delta}^{2}+x(1-x) f\left(\zeta_{l}, y\right)\right]^{1 / 2}}+\frac{8 \alpha}{\tilde{v}_{F}^{2}} \int_{0}^{1} d x \\
& \quad \times\left\{\frac{\tau}{2 \pi} \ln \left[1+2 \cos (2 \pi l x) e^{-g\left(\tau, \zeta_{l}, y\right)}+e^{-2 g\left(\tau, \zeta_{l}, y\right)}\right]-\frac{\zeta_{l}}{2}(1-2 x) \frac{\sin (2 \pi l x)}{\cosh g\left(\tau, \zeta_{l}, y\right)+\cos (2 \pi l x)}\right. \\
& \left.+\frac{\tilde{\Delta}^{2}+\zeta_{l}^{2} x(1-x)}{\left[\tilde{\Delta}^{2}+x(1-x) f\left(\zeta_{l}, y\right)\right]^{1 / 2}} \frac{\cos (2 \pi l x)+e^{-g\left(\tau, \zeta_{l}, y\right)}}{\cosh g\left(\tau, \zeta_{l}, y\right)+\cos (2 \pi l x)}\right\} .
\end{aligned}
$$

Here, $\alpha=e^{2} /(\hbar c) \approx 1 / 137$ is the fine-structure constant, $\tilde{\Delta}=\Delta /\left(\hbar \omega_{c}\right)$ is the dimensionless gap parameter, $\tau=4 \pi a k_{B} T /(\hbar c)=\zeta_{l} / l, \tilde{v}_{F}=v_{F} / c$ is the dimensionless Fermi velocity, the chemical potential $\mu$ is assumed to be equal to zero, and the dimensionless functions $f$ and $g$ are defined as

$$
\begin{aligned}
& f\left(\zeta_{l}, y\right)=\tilde{v}_{F}^{2} y^{2}+\left(1-\tilde{v}_{F}^{2}\right) \zeta_{l}^{2}, \\
& g\left(\tau, \zeta_{l}, y\right)=\frac{2 \pi}{\tau}\left[\tilde{\Delta}^{2}+x(1-x) f\left(\zeta_{l}, y\right)\right]^{1 / 2} .
\end{aligned}
$$

In a similar way, for the sum of two spatial components of the polarization tensor $\tilde{\Pi}_{t r}$ in our dimensionless variables one obtains

$$
\begin{aligned}
& \tilde{\Pi}_{t r}\left(i \zeta_{l}, y\right)=8 \alpha\left[y^{2}+f\left(\zeta_{l}, y\right)\right] \int_{0}^{1} d x \frac{x(1-x)}{\left[\tilde{\Delta}^{2}+x(1-x) f\left(\zeta_{l}, y\right)\right]^{1 / 2}}+\frac{8 \alpha}{\tilde{v}_{F}^{2}} \int_{0}^{1} d x \\
& \quad \times\left\{\frac{\tau}{2 \pi} \ln \left[1+2 \cos (2 \pi l x) e^{-g\left(\tau, \zeta_{l}, y\right)}+e^{-2 g\left(\tau, \zeta_{l}, y\right)}\right]\right. \\
& -\frac{\zeta_{l}\left(1-2 \tilde{v}_{F}^{2}\right)}{2}(1-2 x) \frac{\sin (2 \pi l x)}{\cosh g\left(\tau, \zeta_{l}, y\right)+\cos (2 \pi l x)} \\
& \left.+\frac{\tilde{\Delta}^{2}+x(1-x)\left[\left(1-\tilde{v}_{F}^{2}\right)^{2} \zeta_{l}^{2}-\tilde{v}_{F}^{4} y^{2}\right]}{\left[\tilde{\Delta}^{2}+x(1-x) f\left(\zeta_{l}, y\right)\right]^{1 / 2}} \frac{\cos (2 \pi l x)+e^{-g\left(\tau, \zeta_{l}, y\right)}}{\cosh g\left(\tau, \zeta_{l}, y\right)+\cos (2 \pi l x)}\right\} .
\end{aligned}
$$

It is easily seen that in the limiting case of zero temperature $(T \rightarrow 0, \tau \rightarrow 0)$ we have $g \rightarrow \infty$ and the components of the polarization operator (4) and (6) become much simpler

$$
\begin{aligned}
& \tilde{\Pi}_{00}(i \zeta, y)=\alpha \frac{y^{2}-\zeta^{2}}{f(\zeta, y)} \tilde{\Phi}_{00}(i \zeta, y), \\
& \tilde{\Pi}_{t r}(i \zeta, y)=\alpha \frac{y^{2}+f(\zeta, y)}{f(\zeta, y)} \tilde{\Phi}_{00}(i \zeta, y)
\end{aligned}
$$


where

$$
\tilde{\Phi}_{00}(i \zeta, y)=8 \sqrt{f(\zeta, y)} \int_{0}^{1} d x \frac{x(1-x)}{\left[\frac{\tilde{\Delta}^{2}}{f(\zeta, y)}+x(1-x)\right]^{1 / 2}}
$$

and $\zeta$ is now the continuous dimensionless frequency. Calculating the integral in Eq. (8) we arrive at

$$
\tilde{\Phi}_{00}(i \zeta, y)=4 \tilde{\Delta}+2 \sqrt{f(\zeta, y)}\left[1-4 \frac{\tilde{\Delta}^{2}}{f(\zeta, y)}\right] \arctan \frac{\sqrt{f(\zeta, y)}}{2 \tilde{\Delta}}
$$

After the substitution of Eq. (7) in Eq. (3) the reflection coefficients at zero temperature take the form

$$
\begin{aligned}
& r_{\mathrm{TM}}(i \zeta, y)=\frac{\alpha y \tilde{\Phi}(\zeta, y)}{\alpha y \tilde{\Phi}(\zeta, y)+2 f(\zeta, y)}, \\
& r_{\mathrm{TE}}(i \zeta, y)=-\frac{\alpha \tilde{\Phi}(\zeta, y)}{\alpha \tilde{\Phi}(\zeta, y)+2 y} .
\end{aligned}
$$

These equations were obtained in Ref. [32].

\section{B. Properties of reflection coefficients in the Dirac model}

Now we return to the consideration of general reflection coefficients (3) in the Dirac model with the polarization operator (4) and (6) defined at any nonzero temperature. As can be seen from Eq. (3), for $y=\zeta_{l}$ we have

$$
\left.r_{\mathrm{TM}}\left(i \zeta_{l}, y\right)\right|_{y=\zeta_{l}}=1,\left.\quad r_{\mathrm{TE}}\left(i \zeta_{l}, y\right)\right|_{y=\zeta_{l}}=-1
$$

as it holds for any $\zeta_{l}$ and $y$ for ideal metal plane at both zero and nonzero temperature. At zero temperature, for a graphene sheet described by the Dirac model, Eq. (11), however,

does not hold because in accordance with Eq. (77) the quantity $\left.\tilde{\Pi}_{00}(i \zeta, y)\right|_{y=\zeta}=0$ and both reflection coefficients in (3) become indeterminate form (i.e. zero/zero). The reflection coefficients at $T=0$ are converted to a determinate form in Eq. (10). It is seen that the coefficients $r_{\mathrm{TM}, \mathrm{TE}}(10)$ at $y=\zeta$ are not equal to the limiting values of the coefficients (11) when $T \rightarrow 0$ (i.e., not equal to 1 and -1 , respectively). This means that in the Dirac model the reflection coefficients calculated under the condition $y=\zeta_{l}$ are discontinuous functions of temperature at the point $T=0$ (note that unlike the hydrodynamic model, the reflection coefficients of the Dirac model depend on $T$ not only through the Matsubara frequencies but also explicitly through the polarization operator). 
To illustrate the behavior of the reflection coefficients in the Dirac model, Fig. 1(a) shows by the solid lines $r_{\mathrm{TM}, \mathrm{TE}}\left(i \zeta_{1}, y\right)$ as functions of $y \geq \zeta_{1}=0.49$ at $T=300 \mathrm{~K}, a=300 \mathrm{~nm}$ and $\Delta=0\left(r_{\mathrm{TM}}\right.$ is positive and $r_{\mathrm{TE}}$ is negative). As can be seen in Fig. 1(a), $r_{\mathrm{TM}}$ decreases with decreasing $y$ and abruptly jumps to unity in the vicinity of $y=\zeta_{1}$. In Fig. 1(b) the same is shown in an enlarged scale. The dashed line in Fig. 1(b) shows the coefficient $r_{\mathrm{TM}}\left(i \zeta_{1}, y\right)$ calculated with the polarization operator at zero temperature [i.e., by substituting $\tilde{\Pi}_{00}\left(i \zeta_{1}, y\right)$ from Eq. (7) in Eq. (3) instead of using the operator at $T=300 \mathrm{~K}$ defined in Eq. (4)]. From Fig. 1(b) it is seen that at $l=1$ the use of the polarization operator ta $T=0$ leads to almost the same values of the TM reflection coefficient as the use of the operator at $T=300 \mathrm{~K}$ (the relative difference between the solid and dashed lines for almost all values of $y$ is of about 4\%). Significant difference between the two calculation methods arises only within a very narrow interval from $\zeta_{1}$ to $\zeta_{1}+10^{-7}$. For $l \geq 2$ all differences under discussion become even smaller.

The solid line illustrating the behavior of the reflection coefficient $r_{\mathrm{TE}}\left(i \zeta_{1}, y\right)$ as a function of $y$ in Fig. 1(a) almost coincides with the horizontal coordinate axis in the scales used. In the close proximity of $y=\zeta_{1}$, the reflection coefficient abruptly jumps to minus unity. On an enlarged scale the behavior of $r_{\mathrm{TE}}\left(i \zeta_{1}, y\right)$ as a function of $y$ is shown in Fig. 1(c). From the comparison of Fig. 1(b) and Fig. 1(c), one can conclude that $\mid r_{\mathrm{TE}}\left(i \zeta_{1}, y|\ll| r_{\mathrm{TM}}\left(i \zeta_{1}, y \mid\right.\right.$ for all $y$ with exception of only a very narrow vicinity of the point $y=\zeta_{1}$. Note also that the values of $r_{\mathrm{TE}}\left(i \zeta_{1}, y\right)$ calculated using the polarization operators at zero temperature and at $T=300 \mathrm{~K}$ are indistinguishable in the scales of both Fig. 1(a) and 1(c). The same holds for $l \geq 2$. Thus, the use of the polarization operator (7) instead of (4) and (6) in calculations of $r_{\mathrm{TE}}\left(i \zeta_{1}, y\right)$ with $l \geq 1$ leads to even smaller errors than for $r_{\mathrm{TM}}\left(i \zeta_{1}, y\right)$.

In Ref. [37] it was proposed to use the reflection coefficients (10) taken at zero temperature in all terms of the Lifshitz formula with $l \geq 1$ and restrict the application of the exact reflection coefficients (3), (44) and (6) to only the zero-frequency term $l=0$. In our computations performed below we determine the accuracy of this prescription. 


\section{DEPENDENCE OF THE THERMAL CORRECTION ON A GAP PARAME- TER}

In this section we calculate the free energy of thermal Casimir-Polder atom-graphene interaction and the thermal correction to the Casimir-Polder energy in the framework of the Dirac model of graphene with different values of the gap parameter $\Delta$. The exact value of $\Delta$ is yet unknown. The upper bound on $\Delta$ is approximately equal to $0.1 \mathrm{eV}$, but it might be also much smaller [18]. As an atomic system interacting with graphene, we choose an atom of metastable helium $\mathrm{He}^{*}$ often used in experiments on quantum reflection [17]. To perform computations using the Lifshitz formula (1), one needs the dynamic atomic polarizability of $\mathrm{He}^{*}$ as a function of the imaginary frequency. For many atoms the dynamic polarizability can be expressed with sufficient precision, using the single-oscillator model

$$
\alpha\left(i \omega_{c} \zeta_{l}\right)=\frac{\alpha(0)}{1+\left(\omega_{c}^{2} / \omega_{0}^{2}\right) \zeta_{l}^{2}},
$$

where $\alpha(0)$ is the static polarizability and $\omega_{0}$ is the characteristic absorption frequency. Specifically, for $\mathrm{He}^{*}$ we have $\alpha(0)=\alpha^{\mathrm{He}^{*}}(0)=315.63 \mathrm{a} . \mathrm{u} .=4.678 \times 10^{-29} \mathrm{~m}^{3}$ (where one atomic unit of polarizability is equal to $1.482 \times 10^{-31} \mathrm{~m}^{3}$ ) and $\omega_{0}=\omega_{0}^{\mathrm{He}^{*}}=1.18 \mathrm{eV}=$ $1.794 \times 10^{15} \mathrm{rad} / \mathrm{s}$ [40]. Note that the use of highly accurate dynamic atomic polarizabilities (see, for instance, the polarizability of $\mathrm{He}^{*}$ determined with a relative error $10^{-6}$ [41]) lead to only small relative deviations from the results obtained using Eq. (12). For example, for $\mathrm{He}^{*}$ atom near an $\mathrm{Au}$ wall these deviations decrease from $3.9 \%$ at $a=10 \mathrm{~nm}$ to $0.03 \%$ at $a=1 \mu \mathrm{m}[11,13]$. Our computations show that for graphene the contribution of the term with $l=0$ in Eq. (1) is dominant even at short separations, i.e., the contribution of the static atomic polarizability is of most importance. Because of this, for graphene the single-oscillator model leads to even more accurate results than for metallic walls.

\section{A. Casimir-Polder free energy as a function of temperature}

We have substituted Eqs. (3) $-(6)$ and (12) in Eq. (11) and performed computations of the Casimir-Polder free energy $\mathcal{F}$ as a function of temperature at atom-graphene separation $a=1 \mu \mathrm{m}$ for the values of a gap parameter equal to $\Delta=0.1,0.05,0.01 \mathrm{eV}$ and for $\Delta=0$. The computational results for the quantity $a^{4}|\mathcal{F}|$ are presented in Fig. 2, where the four lines from the lowest to highest correspond to the decreasing values of $\Delta$ (the lowest line 
is for $\Delta=0.1 \mathrm{eV})$. As can be seen in Fig. 2, the characteristic behavior of the free energy differs significantly for different gap parameters. At $T=0$ the values of the Casimir-Polder energy $E(a)=\mathcal{F}(a, 0)$ depend heavily on $\Delta$, whereas at $T=300 \mathrm{~K}$ there is only a slight dependence of the Casimir-Polder free energy $\mathcal{F}(a, T)$ on $\Delta$. This allows estimation of $\Delta$ from the comparison between the measurement data and computational results in the region of moderate temperatures from 100 to $150 \mathrm{~K}$. Furthermore, the larger is $\Delta$, the wider is the temperature region where $\mathcal{F}$ remains constant with the increase of temperature. In such temperature regions the thermal correction to the Casimir-Polder energy is negligibly small. Below we discuss this point in more detail.

To check quantitatively an accuracy of the prescription [37] that in all terms of the Lifshitz formula with $l \geq 1$ one can use the polarization operator at $T=0$, we repeated the same computation as above, but this time with the operators (44) and (6) for $l=0$ and (7) for all $l \geq 1$. The obtained results cannot be distinguished visually from those shown in Fig. 2. The largest deviations between the computational results obtained using different calculation procedures (with the polarization operators found at $T \neq 0$ for all $l$ and for only $l=0$ ) hold with the gap parameter $\Delta=0$. In this case, the magnitudes of the free energy obtained using the prescription are smaller than those in full computations by $0.6 \%, 0.3 \%$, $0.1 \%$, and $0.06 \%$ at $T=300 \mathrm{~K}$ and separations $a=50,200,500$, and $1000 \mathrm{~nm}$, respectively. At $a=3 \mu \mathrm{m}$ the relative difference between the computational results obtained using the two procedures is as small as $0.002 \%$. One can conclude that the prescription of Ref. [37] leads to very accurate results and can be used in subsequent computations.

\section{B. Thermal correction as a function of separation}

Now we calculate the thermal correction to the Casimir-Polder energy in the interaction of He* atom with graphene as a function of separation. The relative thermal correction at a temperature $T$ is defined as

$$
\delta_{T} \mathcal{F}(a, T)=\frac{\mathcal{F}(a, T)-\mathcal{F}(a, 0)}{\mathcal{F}(a, 0)} .
$$

The computations are performed at $T=300 \mathrm{~K}$ using Eq. (1) with full polarization operator

for $l=0$ and zero-temperature operators for $l \geq 1$. The computational results within the separation region from 10 to $500 \mathrm{~nm}$ are shown in Fig. 3(a) by the six solid lines from the 
highest to the lowest corresponding to the values of the gap parameter $\Delta=0.1,0.05,0.025$, $0.01,0.001$, and $0 \mathrm{eV}$, respectively. Note that the two lowest lines obtained for two smallest values of the gap parameter are almost coinciding. It is interesting that at $T=300 \mathrm{~K}$ the magnitude of the relative thermal correction at each separation increases monotonically with increasing $\Delta$. The largest thermal correction is achieved for $\Delta=0.1 \mathrm{eV}$. At $T=300 \mathrm{~K}$ the same properties hold at shorter separation distances below $100 \mathrm{~nm}$. This separation region is ahown in an enlarged scale in Fig. 3(b).

As suggested by Fig. 2, the monotonous dependence of the relative thermal correction on $\Delta$ is not universal and does not hold at any temperature. As an example we have computed the thermal correction to the energy of $\mathrm{He}^{*}$-graphene interaction at $T=100 \mathrm{~K}$. This is the maximum temperature until which the free energy is nearly constant for $\Delta=0.1 \mathrm{eV}$. The computational results within the separation region from 10 to $500 \mathrm{~nm}$ are shown in Fig. 4(a) by the three solid lines from the lowest to the highest corresponding to the following values of the gap parameter: $\Delta=0.1,0.05$, and $0.025 \mathrm{eV}$. As is seen in Fig. $4(\mathrm{a})$, for $\Delta=0.1 \mathrm{eV}$ the thermal correction is very small over the entire separation region. This is in accordance with the computational results shown in Fig. 2. The computational results for the same thermal correction at $T=100 \mathrm{~K}$, but with the values of the gap parameter equal to $\Delta=0.025,0.01$, 0.001, and $0 \mathrm{eV}$, are shown in Fig. 4(b) by the solid lines from the highest to the lowest line, respectively. For illustration purposes, the line with $\Delta=0.025 \mathrm{eV}$ is reproduced in both Fig. 4(a) and 4(b). From the comparison of Fig. 4(a) with Fig. 4(b) one can see that at $T=100 \mathrm{~K}$ the dependence of the thermal correction on $\Delta$ is not monotonous. From Fig. 4(b) it can be seen also that there is a noticeable difference between the thermal corrections for a graphene with $\Delta=0.001 \mathrm{eV}$ and with $\Delta=0$. Similar results are obtained for other atoms.

One can conclude that the size of thermal correction to the Casimir-Polder interaction of an atom with a graphene sheet depends essentially on the size of the gap in the spectrum of graphene quasiparticles. From our computations it follows that if the inequality $k_{B} T \ll \Delta$ is satisfied with a large safety margin, then the relative thermal correction is small (note that $T=100 \mathrm{~K}$ corresponds to $k_{B} T=0.0083 \mathrm{eV}$ to be compared with $\left.\Delta=0.1 \mathrm{eV}\right)$. On the contrary, if $\Delta \lesssim k_{B} T$, then the thermal correction is large (in so doing, the thermal correction can be also large for $k_{B} T$ smaller but not much smaller than $\Delta$ ). Keeping in mind that the value of $\Delta$ for graphene is not yet known [18], the predictions of the Dirac model concerning the size of thermal correction in atom-graphene interaction remain uncertain. 


\section{DISTANCE DEPENDENCE FOR THE CASIMIR-POLDER INTERACTION OF DIFFERENT ATOMS WITH GRAPHENE}

We come now to the Casimir-Polder interaction of graphene with atoms of $\mathrm{He}^{*}$, $\mathrm{Na}$, $\mathrm{Rb}$, and $\mathrm{Cs}$ at room temperature $T=300 \mathrm{~K}$ but at different separation distances, as is of interest for experiments on quantum reflection. Here, we compare the computational results obtained using both the hydrodynamic and Dirac models for graphene.

\section{A. Atom of metastable helium}

We start with an atom of $\mathrm{He}^{*}$ and compute the Casimir-Polder free energy (11) with the reflection coefficients (2) of the hydrodynamic model. The computational results for $a^{4}|\mathcal{F}|$ as a function of separation from $50 \mathrm{~nm}$ to $5 \mu \mathrm{m}$ are presented in Fig. 5(a) by the dashed line. Then we repeated computations using Eq. (1), but with the Dirac reflection coefficients (3), which contain the polarization operator (44), (6) obtained at $T=300 \mathrm{~K}$ [as was noted in Sec. II, it is sufficient to use this operator only with $l=0$ term and replace it by a more simple operator (7) in all terms with $l \geq 1$ ]. In this case the computational results for the gap parameter in the region from 0 to $0.01 \mathrm{eV}$ are shown by the solid line. Note that even the use of larger gap parameters up to $0.1 \mathrm{eV}$ leads to almost imperceptible shift of the solid line in Fig. 5(a). This is explained by the fact that at $T=300 \mathrm{~K}$ there are only minor differences between the Casimir-Polder free energies computed with different $\Delta$ (see Fig. 2).

In Fig. 5(a)we also plot by the dotted line the computational results obtained using the Lifshitz formula (11) and the Dirac model with $\Delta=0$ at zero temperature [i.e., using the polarization operator (17) with all Matsubara terms with $l \geq 0$ ]. As can be seen from Fig. 5(a), the computational results shown by the solid line are by factors of $1.27,1.29$, and 1.29 larger than those shown by the dotted line at separations 1,3 , and $5 \mu \mathrm{m}$, respectively. This confirms that in order to perform precise computations at $T=300 \mathrm{~K}$, one should use the polarization operator at the same temperature (at least in the zero-frequency contribution of the Lifshitz formula). From Fig. 5(a) it is also seen that the predictions of the hydrodynamic model at the separations $0.2,0.5,1.0,1.5,2$, and $3 \mu \mathrm{m}$ (the dashed line) are by factors of 3.83, 2.96, 2.07, 1.62, 1.36, and 1.12 larger than the predictions of the Dirac model (the solid line). This allows reliable discrimination between theoretical predictions of the two models 
by the measurement data of experiments on quantum reflection. (Note that in Ref. [33], where computations were performed at $a<100 \mathrm{~nm}$ using the polarization operator at $T=0$, the predictions of the Dirac model were overestimated by approximately a factor of 1.5 due to an error in the computer program. As a result, the differences between the predictions of two models at short separations were underestimated.)

From the comparison of solid and dotted lines in Fig. 5(a), we have already found errors arising from the use of polarization operator at $T=0$ in all terms of the Lifshitz formula (an underestimation of $|\mathcal{F}|$ by approximately a factor of 1.3). This conclusion was obtained, however, from the zero-temperature polarization operator with $\Delta=0$ (as was demonstrated above, computational results with the $T$-dependent polarization operator are not sensitive to the value of $\Delta$ at $T=300 \mathrm{~K}$ ). To investigate the role of the gap parameter in computations using the polarization operator at $T=0$, in Fig. 5(b) we plot $a^{4}|\mathcal{F}|$ as a function of separation computed with the full operator (44), (6) (the solid line) and with the operator (7) for $\Delta=0,0.001$, and $0.01 \mathrm{eV}$ (the dotted lines from the highest to the lowest, respectively). Note that the solid and the highest dotted lines reproduce the respective lines in Fig. 5(a) at separations from $50 \mathrm{~nm}$ to $1 \mu \mathrm{m}$. As can be seen from Fig. 5(b), for nonzero $\Delta$ the underestimation of $|\mathcal{F}|$ when using the polarization operator at $T=0$ is much larger than for $\Delta=0$. Thus, at $a=1 \mu \mathrm{m}$ this underestimation is by the factors of 1.83 and 6.2 for the gap parameter $\Delta=0.001$ and $0.01 \mathrm{eV}$, respectively. This adds importance to the use of full temperature-dependent polarization operator (44), (6) in computations performed for subsequent comparison with the experimental data.

\section{B. Atoms of sodium, rubidium and cesium}

The Casimir-Polder free energy for other atoms used in experiments on quantum reflection can be computed in a similar way. For Na the dynamic polarizability can be presented by Eq. (12) with $\alpha^{\mathrm{Na}}(0)=162.68$ a.u. $=2.411 \times 10^{-29} \mathrm{~m}^{3}$ and $\omega_{0}^{\mathrm{Na}}=2.14 \mathrm{eV}=3.25 \times$ $10^{15} \mathrm{rad} / \mathrm{s}$ [42]. The computational results for the quantity $a^{4}|\mathcal{F}|$ for Na-graphene interaction at $T=300 \mathrm{~K}$ are presented in Fig. 6(a) as function of separation by the dashed line (the hydrodynamic model), the solid line [the Dirac model with the $T$-dependent polarization operator (44), (6)], and by the dotted line [the Dirac model with the polarization operator (7) at $T=0, \Delta=0]$. It can be seen that qualitatively the computational results in Fig. 6(a) 
are similar to those in Fig. 5(a) for a $\mathrm{He}^{*}$ atom. In the case of $\mathrm{Na}$, however, the values of $a^{4}|\mathcal{F}|$ are smaller than for $\mathrm{He}^{*}$ at all respective separations. The difference between the predictions of the hydrodynamic and Dirac models can be easily discriminated by the measurement data of experiments on quantum reflection. Thus, at separations $0.2,0.5,1.0$, 1.5, and $2 \mu \mathrm{m}$ the predictions of the hydrodynamic model are larger than the predictions of the Dirac model with the $T$-dependent polarization operator by factors of 4.04, 3.05, $2.10,1.63$, and 1.37 , respectively. The use of the polarization operator at $T=0$ again underestimates the predictions of the Dirac model (by the factors of 1.27, 1.29, and 1.29 at separations $a=1,3$, and $5 \mu \mathrm{m}$, respectively).

In Figs. 6(b) and 6(c) similar results are presented for atoms Rb and Cs interacting with graphene. To perform computations of the Casimir-Polder free energy using different models of graphene, we have used the single-oscillator model (12) with the following parameters: $\alpha^{\mathrm{Rb}}(0)=319.9$ a.u. $=4.73 \times 10^{-29} \mathrm{~m}^{3}, \omega_{0}^{\mathrm{Rb}}=5.46 \mathrm{eV}=8.3 \times 10^{15} \mathrm{rad} / \mathrm{s}[43]$ and $\alpha^{\mathrm{Cs}}(0)=$ 403.6 a.u. $=5.981 \times 10^{-29} \mathrm{~m}^{3}$ and $\omega_{0}^{\mathrm{Cs}}=1.55 \mathrm{eV}=2.36 \times 10^{15} \mathrm{rad} / \mathrm{s} \mathrm{[42,} \mathrm{44]}$. As can be seen from the comparison of Fig. 6(b) with Fig. 6(a) and Fig. 6(c) with Fig. 6(b), the magnitudes of the free energy for $\mathrm{Rb}$ are larger than for $\mathrm{Na}$ and the respective magnitudes for Cs are larger than for Rb at all separation distances. This is explained by the fact that the static polarizability of Cs is the largest one. From Figs. 6(b) and 6(c) one can observe the same pattern between the dashed, solid and dotted lines, obtained for the hydrodynamic model and for the Dirac model with $T$-dependent and $T$-independent polarization operators, respectively, as was discussed on the basis of Figs. 5(a) and 6(a).

In Figs. 5(a) and 6(a-c), the distinction between the predictions of the hydrodynamic model (the dashed lines) and the Dirac model with $T$-dependent polarization operator (the solid lines) deserves special attention. The point is that the dashed lines demonstrate the same qualitative behavior [15] as the Casimir-Polder free energy of atoms interacting with walls made of ordinary real materials, such as $\mathrm{Au}$ or Si. By contrast, the Dirac model for graphene predicts quite a different behavior of the free energy, where it is nearly inverse proportional to the third power of separation. Keeping in mind that at separations below $2 \mu \mathrm{m}$ the predictions of both models may differ by a factor of 2 and even up to a factor of 4 , it seems appropriate to raise a question which model is in better agreement with the experimental data. This question can be answered through experiments on quantum reflection of different atoms on suspended graphene membranes. 


\section{CONCLUSIONS AND DISCUSSION}

In this work we have investigated the Casimir-Polder interaction of different atoms with graphene in the thermal regime. Special attention was paid to the Dirac model of graphene and to the conditions when thermal effects become essential. We have confirmed the conclusion [37] that sufficient precision can be achieved by using the temperature-dependent polarization operator in the zero-frequency term of the Lifshitz formula alone and calculating all the other terms using the polarization operator at $T=0$. We have found that a major impact on the thermal correction (i.e. whether it is small or large) is made by the size of the gap $\Delta$ in the spectrum of graphene nanoparticles. According to our results, if the condition $k_{B} T \ll \Delta$ is satisfied with a large safety margin, the thermal correction to the Casimir-Polder interaction of an atom with graphene is relatively small. In future it is planed to obtain the asymptotic behavior of the free energy under this condition analytically and to verify the fulfilment of the Nernst heat theorem in the Dirac model. If, however, $\Delta \lesssim k_{B} T$, the thermal correction is relatively large.

Furthermore, we have calculated the free energy of the Casimir-Polder interaction of different atoms used in experiments on quantum reflection (such as $\mathrm{He}^{*}, \mathrm{Na}, \mathrm{Rb}$, and $\mathrm{Cs}$ ) with a suspended graphene membrane. All computations were performed for room temperature within a wide separation region in the framework of two models for graphene proposed in the literature: the hydrodynamic and Dirac ones. It is important to note that both these models are approximations and a priori it is not possible to decide which of them provides a better theoretical description of the Casimir-Polder force (for example, the Drude model of metals describes correctly the electric properties of metals and their dielectric response to external electromagnetic fields, but is in disagreement with the experimental data on measurements of the Casimir force between metallic test bodies [6, 30]).

Our computational results allow to conclude that the hydrodynamic and Dirac models of graphene lead to both qualitatively and quantitatively different results for the free energy of atom-graphene Casimir-Polder interaction. On the qualitative side, we have arrived at quite different dependences of the free energy on separation distance. Quantitatively, the magnitudes of the free energies computed using the two models differ by a factor of 2 and even by a factor of 4 at different separations below $2 \mu \mathrm{m}$. A difference in theoretical predictions being as large as by this amount assures a reliable discrimination between the 
hydrodynamic and Dirac models of graphene by comparing with the experimental data on quantum reflection. Keeping in mind that suspended graphene membranes have been

already produced [39], one could expect the resolution of this problem in the immediate future.

\section{Acknowledgments}

The financial support of the Academy of Finland under the Projects No. 136539 and 140886 is gratefully acknowledged. G.L.K. and V.M.M. were also partially supported by the DFG grant BO 1112/21-1.

[1] F. London, Z. Phys. 63, 245 (1930).

[2] H. B. G. Casimir and D. Polder, Phys. Rev. 73, 360 (1948).

[3] I. E. Dzyaloshinskii, E. M. Lifshitz, and L. P. Pitaevskii, Adv. Phys. 38, 165 (1961) [Usp. Fiz. Nauk 73, 381 (1961)].

[4] J. Mahanty and B. W. Ninham, Dispersion Forces (Academic Press, London, 1976).

[5] V. A. Parsegian, Van der Waals Forces: A Handbook for Biologists, Chemists, Engineers, and Physicists (Cambridge University Press, Cambridge, 2005).

[6] M. Bordag, G. L. Klimchitskaya, U. Mohideen, and V. M. Mostepanenko, Advances in the Casimir Effect (Oxford University Press, Oxford, 2009).

[7] F. Shimizu, Phys. Rev. Lett. 86, 987 (2001).

[8] V. Druzhinina and M. DeKieviet, Phys. Rev. Lett. 91, 193202 (2003).

[9] Y. Lin, I. Teper, C. Chin, and V. Vuletić, Phys. Rev. Lett. 92, 050404 (2004).

[10] B. Segev, R. Côté, and M. G. Raizen, Phys. Rev. A 56, R3350 (1997).

[11] J. F. Babb, G. L. Klimchitskaya, and V. M. Mostepanenko, Phys. Rev. A 70, 042901 (2004).

[12] M. Antezza, L. P. Pitaevskii, and S. Stringari, Phys. Rev. A 70, 053619 (2004).

[13] A. O. Caride, G. L. Klimchitskaya, V. M. Mostepanenko, and S. I. Zanette, Phys. Rev. A 71, 042901 (2005).

[14] S. Y. Buhmann and D.-G. Welsch, Progr. Quant. Electronics 31, 51 (2007). 
[15] V. B. Bezerra, G. L. Klimchitskaya, V. M. Mostepanenko, and C. Romero, Phys. Rev. A 78, 042901 (2008).

[16] H. Friedrich, G. Jacoby, and C. G. Meister, Phys. Rev. A 65, 032902 (2002).

[17] H. Oberst, Y. Tashiro, K. Shimizu, and F. Shimizu, Phys. Rev. A 71, 052901 (2005).

[18] A. H. Castro Neto, F. Guinea, N. M. R. Peres, K. S. Novoselov, and A. K. Geim, Rev. Mod. Phys. 81, 109 (2009).

[19] A. Bogicevic, S. Ovesson, P. Hyldgaard, B. I. Lundqvist, H. Brune, and D. R. Jennison, Phys. Rev. Lett. 85, 1910 (2000).

[20] E. Hult, P. Hyldgaard, J. Rossmeisl, and B. I. Lundqvist, Phys. Rev. B 64, 195414 (2001).

[21] J. Jung, P. García-González, J. F. Dobson, and R. W. Godby, Phys. Rev. B 70, 205107 (2004).

[22] J. F. Dobson, A. White, and A. Rubio, Phys. Rev. Lett. 96, 073201 (2006).

[23] I. V. Bondarev and Ph. Lambin, Phys. Rev. B 70, 035407 (2004).

[24] E. V. Blagov, G. L. Klimchitskaya, and V. M. Mostepanenko, Phys. Rev. B 71, 235401 (2005).

[25] S. Y. Buhmann, S. Scheel, S. Å. Ellingsen, K. Hornberger, and A. Jacob, Phys. Rev. A 85, $042513(2012)$.

[26] G. Barton, J. Phys. A 37, 1011 (2004).

[27] G. Barton, J. Phys. A 38, 2997 (2005).

[28] M. Bordag, B. Geyer, G. L. Klimchitskaya, and V. M. Mostepanenko, Phys. Rev. B 74, 205431 (2006).

[29] E. V. Blagov, G. L. Klimchitskaya, and V. M. Mostepanenko, Phys. Rev. B 75, 235413 (2007).

[30] G. L. Klimchitskaya, U. Mohideen, and V. M. Mostepanenko, Rev. Mod. Phys. 81, 1827 (2009).

[31] A. K. Geim and K. S. Novoselov, Nature Mater. 6, 183 (2007).

[32] M. Bordag, I. V. Fialkovsky, D. M. Gitman, and D. V. Vassilevich, Phys. Rev. B 80, 245406 (2009).

[33] Yu. V. Churkin, A. B. Fedortsov, G. L. Klimchitskaya, and V. A. Yurova, Phys. Rev. B 82, $165433(2010)$.

[34] T. A. Pasquini, M. Saba, G. Jo, Y. Shin, W. Ketterle, D. E. Pritchard, T. A. Savas, and N. Mulders, Phys. Rev. Lett. 97, 093201 (2006).

[35] T. A. Pasquini, Y. Shin, C. Sanner, M. Saba, A. Schirotzek, D. E. Pritchard, and W. Ketterle, Phys. Rev. Lett. 93, 223201 (2004). 
[36] G. Gómez-Santos, Phys. Rev. B 80, 245424 (2009).

[37] I. V. Fialkovsky, V. N. Marachevsky, and D. V. Vassilevich, Phys. Rev. B 84, 035446 (2011).

[38] T. E. Judd, R. G. Scott, A. M. Martin, B. Kaczmarek, and T. M. Fromhold, New. J. Phys. 13, 083020 (2011).

[39] B. Alemán, W. Regan, S. Aloni, V. Altoe, N. Alem, C. Girit, B. Geng, L. Maserati, M. Crommie, F. Wang, and A. Zettl, ACS Nano 4, 4762 (2010).

[40] R. Brühl, P. Fouquet, R. E. Grisenti, J. P. Toennies, G. C. Hegerfeldt, T. Köhler, M. Stoll, and C. Walter, Europhys. Lett. 59, 357 (2002).

[41] Z.-C. Yan and J. F. Babb, Phys. Rev. A 58, 1247 (1998).

[42] A. Derevianko, W. R. Johnson, M. S. Safronova, and J. F. Babb, Phys. Rev. Lett. 82, 3589 (1999).

[43] M. S. Safronova, C. J. Williams, and C. W. Clark, Phys. Rev. A 69, 022509 (2004).

[44] A. Derevianko and S. G. Porsev, Phys. Rev. A 65, 053403 (2002). 

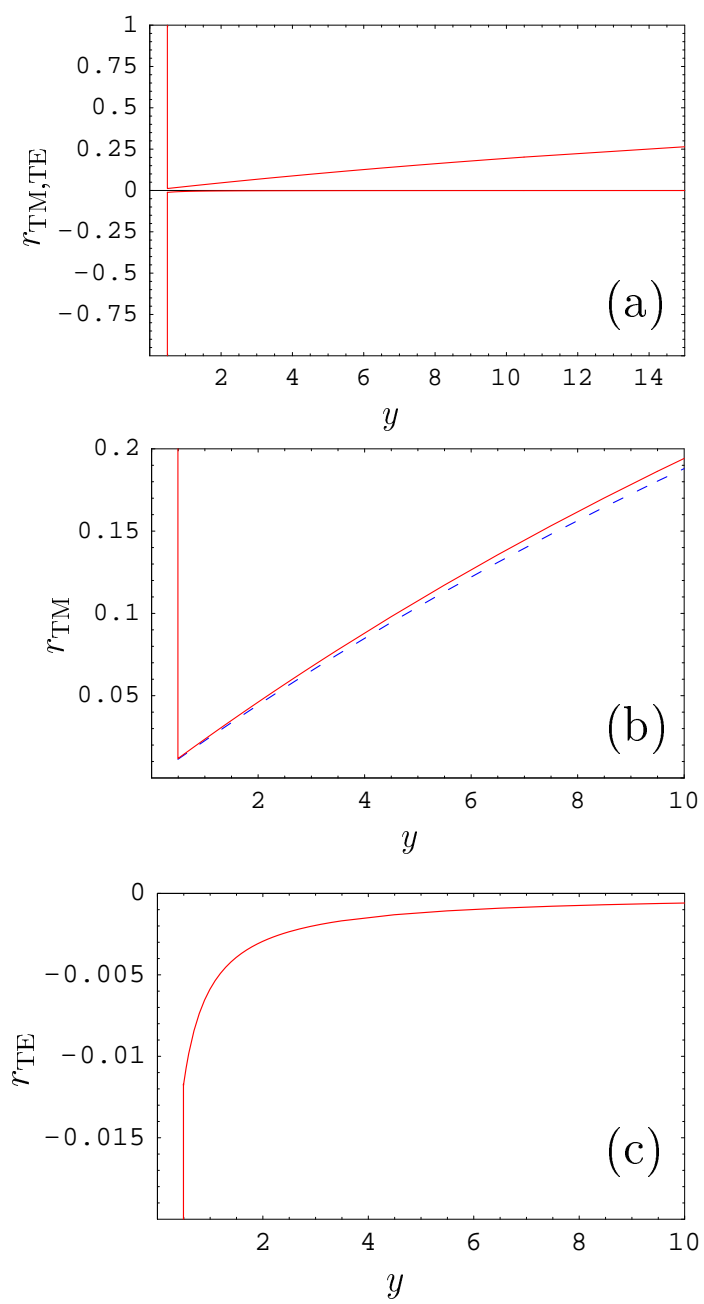

FIG. 1: (Color online) (a) The reflection coefficients $r_{\mathrm{TM}, \mathrm{TE}}$ on graphene calculated at $\zeta=\zeta_{1}$, $T=300 \mathrm{~K}$ and $a=300 \mathrm{~nm}$ as functions of the dimensionless variable $y$ using the polarization tensor at $T=300 \mathrm{~K}$ are shown by the positive- and negative-valued solid lines, respectively. (b) The solid and dashed lines show $r_{\mathrm{TM}}$ computed using the polarization tensor at $T=300 \mathrm{~K}$ and $T=0$, respectively. (c) The solid line shows $r_{\mathrm{TE}}$. The scales in (b) and (c) are enlarged as compared to (a). 


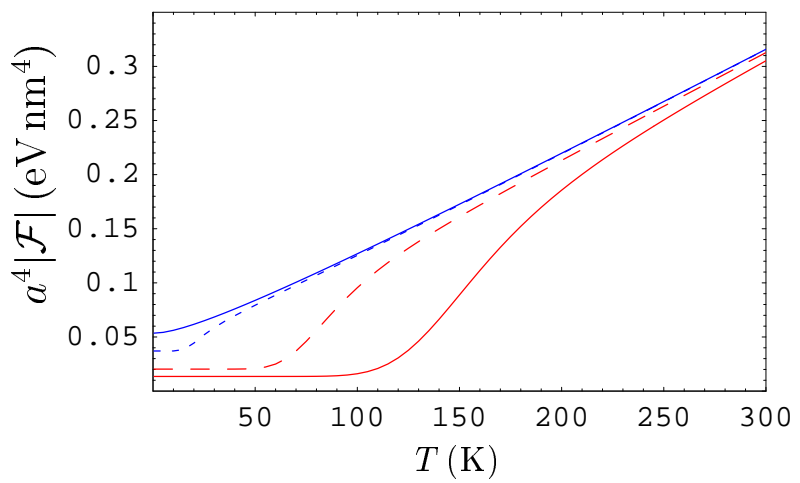

FIG. 2: (Color online) The Casimir-Polder free energy of He*-graphene interaction at $a=1 \mu \mathrm{m}$ multiplied by the fourth power of separation is shown as a function of temperature by the four lines from the lowest to the highest for the gap parameter $\Delta=0.1,0.05,0.01$, and $0 \mathrm{eV}$, respectively. 

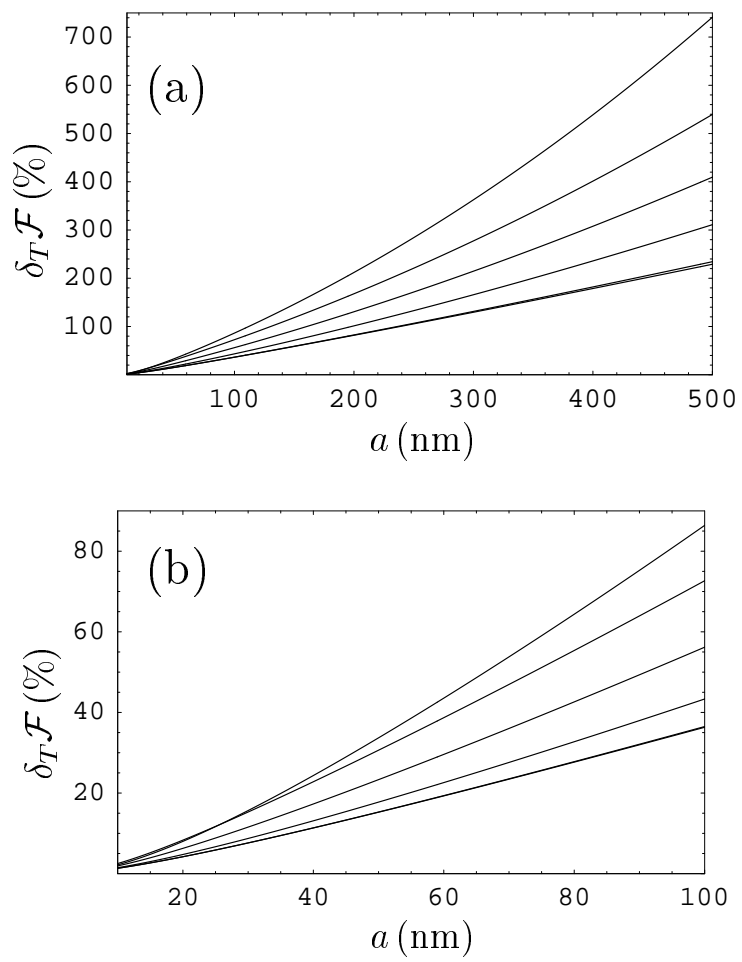

FIG. 3: (a) The relative thermal correction to the Casimir-Polder energy of He*-graphene interaction at $T=300 \mathrm{~K}$ multiplied by the fourth power of separation is shown as a function of separation by the solid lines from the highest to the lowest for the gap parameter $\Delta=0.1,0.05,0.025,0.01$, 0.001, and $0 \mathrm{eV}$, respectively. (b) The same is shown at separations from 10 to $100 \mathrm{~nm}$. 

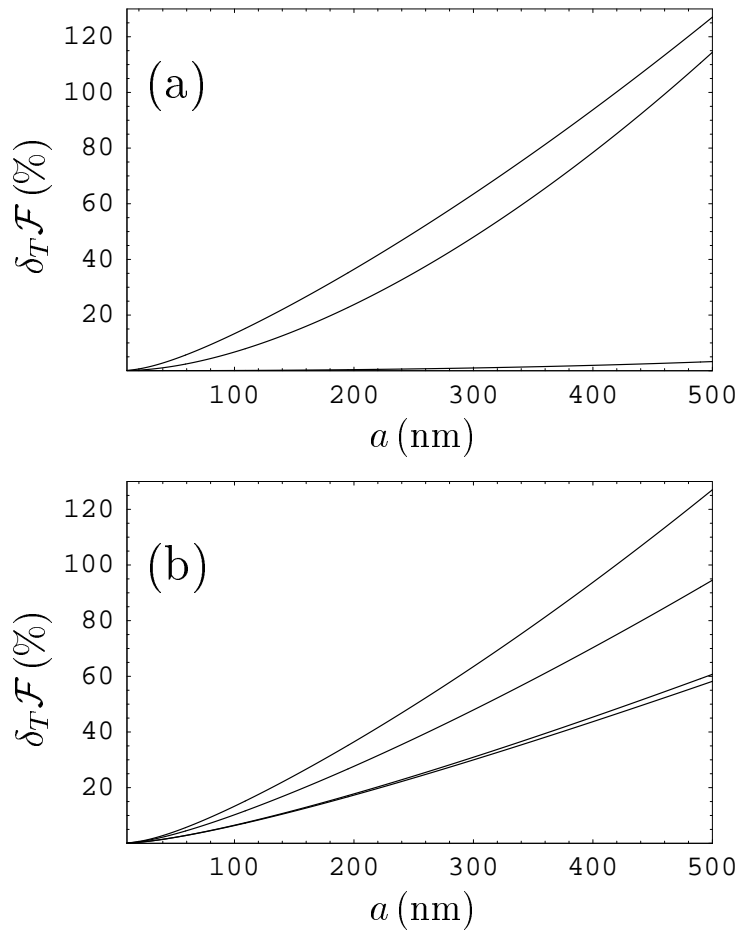

FIG. 4: The relative thermal correction to the Casimir-Polder energy of He*-graphene interaction at $T=100 \mathrm{~K}$ multiplied by the fourth power of separation is shown as a function of separation by the solid lines (a) from the lowest to the highest for the gap parameter $\Delta=0.1,0.05$, and $0.025 \mathrm{eV}$ and (b) from the highest to the lowest for the gap parameter $\Delta=0.025,0.01,0.001$, and $0 \mathrm{eV}$. 

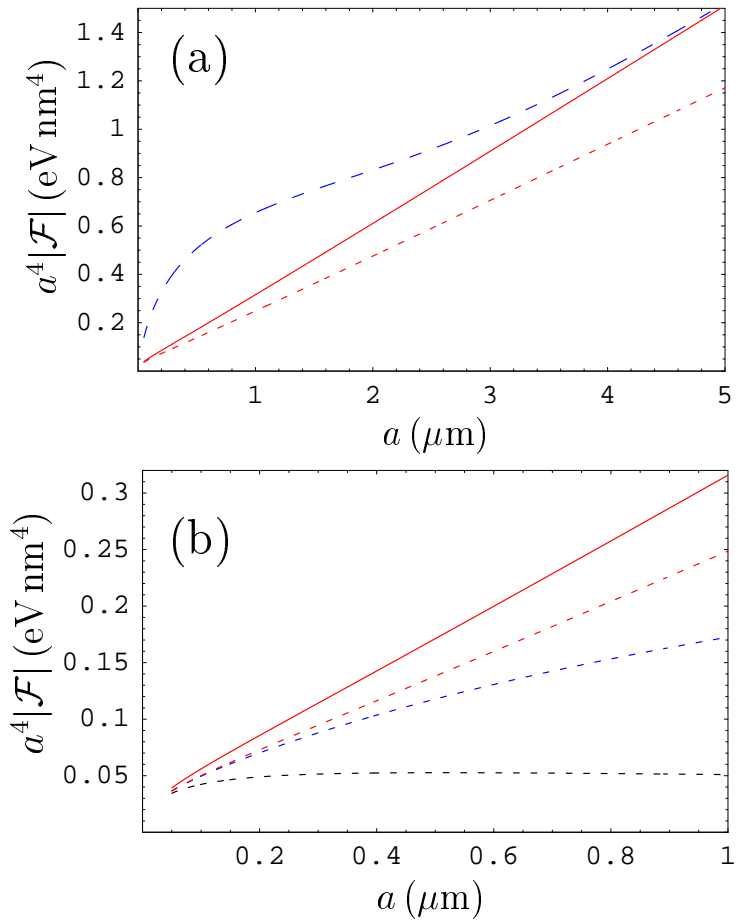

FIG. 5: (Color online) The Casimir-Polder free energy of $\mathrm{He}^{*}$-graphene interaction at $T=300 \mathrm{~K}$ multiplied by the fourth power of separation is shown as a function of separation (a) by the dashed, solid and dotted lines using the hydrodynamic model, the Dirac model with $T$-dependent polarization operator, and with the polarization operator at zero temperature, respectively. (b) The same quantity is shown where the dotted lines from the highest to the lowest are computed using the polarization operator at zero temperature with the gap parameter $\Delta=0,0.001$, and $0.01 \mathrm{eV}$, respectively. 

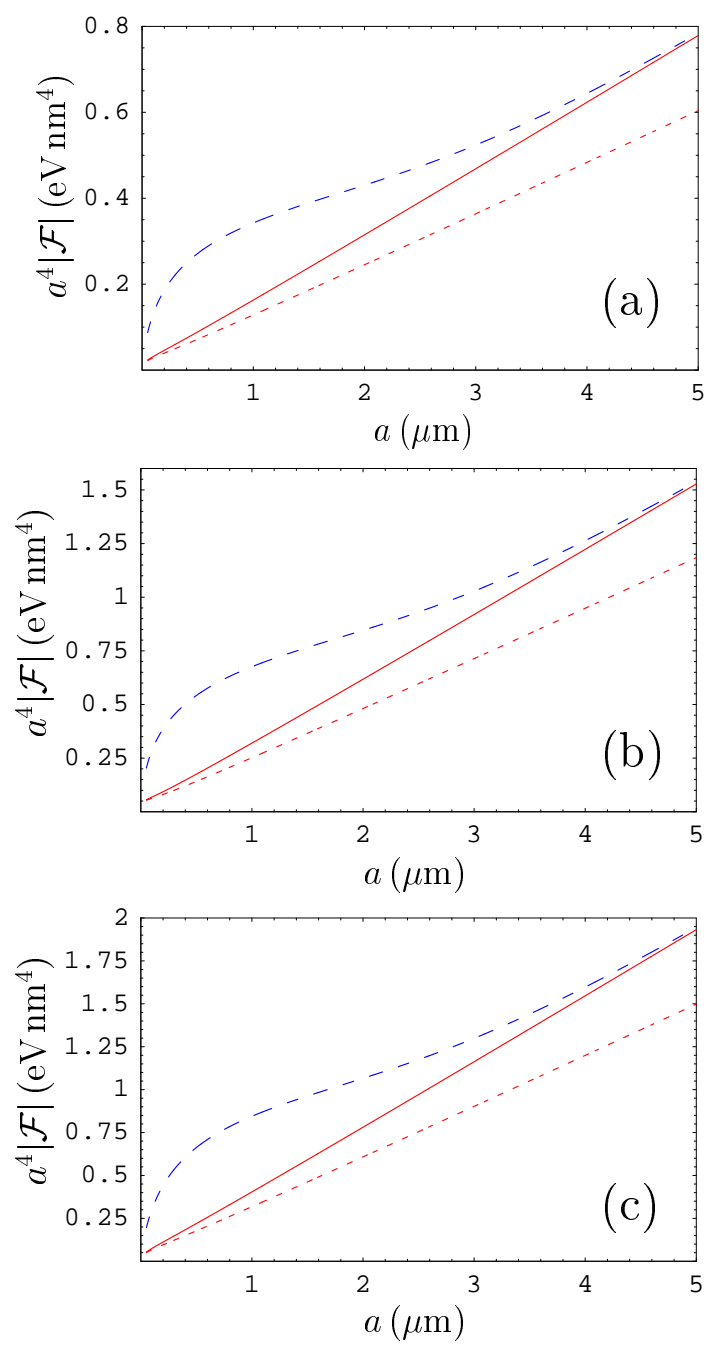

FIG. 6: (Color online) The Casimir-Polder free energy of atom-graphene interaction at $T=300 \mathrm{~K}$ multiplied by the fourth power of separation is shown as a function of separation by the dashed, solid and dotted lines using the hydrodynamic model, the Dirac model with $T$-dependent polarization operator, and with the polarization operator at zero temperature, respectively, for atoms of (a) $\mathrm{Na}$, (b) Rb, and (c) Cs. 